\title{
Distinct characterization of two vinorelbine-resistant breast cancer cell lines developed by different strategies
}

\author{
MINGJIE XU, DONGHAI JIANG, JIAYING SHEN, HUILIN ZHENG and WEIMIN FAN \\ Program of Innovative Cancer Therapeutics, The First Affiliated Hospital of \\ Zhejiang University School of Medicine, Hangzhou, Zhejiang, P.R. China
}

Received November 6, 2015; Accepted December 17, 2015

DOI: $10.3892 /$ or.2016.4566

\begin{abstract}
Resistance to chemotherapy is a major obstacle to the successful treatment of breast cancer patients. Recently, we successfully established two vinorelbine-resistant sublines, BC-DS and BC-TS, from the human breast cancer cell line BCap37, with different 'two-stage screening methods'. Interestingly, though BC-DS and BC-TS were developed from the same BCap37 cell line with the same drug, they showed remarkable differences. Compared with the parental BCap37 cells both BC-DS and BC-TS had resistance to vinorelbine, but the resistant characterizations are both unstable. BC-DS showed increased migration capability while BC-TS showed reduced migration capability. When investigating their multidrug resistance, we found $\mathrm{BC}-\mathrm{DS}$ became more sensitive to methotrexate, which suggested that combination of MTX and vinorelbine could be a new treatment strategy. Moreover, BC-DS and BC-TS overexpressed P-glycoprotein at different levels. Our research also showed that the present clinical usage of vinorelbine is reasonable. These findings suggest that the vinorelbine-induced multiple drug resistance (MDR) sublines may be used as an in vitro model not only to further elucidate possible mechanisms of MDR involved in the human breast cancer, but also to find methods to optimize the curative effect of vinorelbine in clinic.
\end{abstract}

\section{Introduction}

Breast cancer is the most frequently diagnosed cancer and the leading cause of cancer death in female worldwide, accounting for $23 \%$ (1.38 million) of the total new cancer cases and $14 \%$

Correspondence to: Professor Weimin Fan, Program of Innovative Cancer Therapeutics, The First Affiliated Hospital of Zhejiang University School of Medicine, Hangzhou, Zhejiang, P.R. China E-mail: fanw@zju.edu.cn

Abbreviations: VNB, vinorelbine; BC-DS, BCap37 dose stimulated; BC-TS, BCap37 time stimulated; MDR, multiple drug resistance; P-gp, P-glycoprotein; MTX, methotrexate

Key words: two-stage screening method, P-glycoprotein, breast cancer, drug resistance, vinorelbine
$(458,400)$ of the total cancer deaths in 2008 (1). At present, chemotherapy is one of the main methods to treat cancer, and drug-resistance is the main obstacle to ensure optimal outcomes. So drug-resistance is one of the key blocks on the way to cure cancer.

Vinorelbine (Navelbine ${ }^{\circledR}$ ) is a semisynthetic vinca alkaloid that interferes with microtubule assembly and induces a cell cycle arrest at mitosis due to its microtubule targeting activity. Vinorelbine has a broad spectrum of antitumor activity (2), it has been shown to be active as a single agent in metastatic breast cancer, with response rates of $40-60 \%$ in chemonaive disease and with good tolerability (3). Vinorelbine tends to be reserved for anthracycline-resistant disease in the second- or third-line therapy. Although drug resistance is common in the second- or third-line therapy, there are few studies of vinorelbine-resistant breast cancer.

To illustrate the mechanism of drug resistance, many research teams have successfully established a variety of drug-resistant tumor cell lines (4-7). Currently, the most typical method to establish a drug-resistant tumor cell line in vitro is stimulating tumor cells with a certain concentration of chemotherapy drug continuously while increasing the concentration gradually (8-10), but some studies state that this method has limitations $(11,12)$. As the purpose to establish a drug-resistant tumor cell line is to provide a proper research tool for overcoming drug-resistance in clinic, the method should imitate the clinical chemotherapy setting, which commonly contains several cycles of 21 to 28 days discontinuous administration $(13,14)$.

In this study, two vinorelbine-resistant breast cancer cell lines, BC-DS (BCap37-dose stimulated) and BC-TS (BCap37-time stimulated), were developed from the chemo-sensitive human breast cancer cell line BCap37 by different screening strategies. By investigating their biological characterization and drug-resistant traits, we found differences between them in different levels. This indicates that different drug-resistant cell lines can be developed with a certain drug even from the same cell line.

\section{Materials and methods}

Cell lines and mice. Human breast cancer cell lines BCap37, BC-DS and BC-TS, were cultured in RPMI-1640 medium supplemented with $10 \%$ fetal bovine serum (FBS), and frozen 
in liquid nitrogen. After thawing, experiments were done within two weeks. Female, aged 5-6 weeks, athymic nude (nu/nu) mice were purchased from Shanghai SLAC Animal Facility. All animal care and experiments were conducted according to Zhejiang University Animal Care Committee guidelines.

Observation of morphology under microscope. BCap37, BC-DS and BC-TS cells were sub-cultured into 6-cm dishes for $48 \mathrm{~h}$ to reach logarithmic growth phase. Cells were first observed and photographed under an inverted microscope. Then Giemsa staining (Jiangcheng Biotech, Nanjing, China) was carried out.

Cell growth rate in vitro. Cell lines was plated into ten $6-\mathrm{cm}$ dishes with a density of $4 \times 10^{4}$ cells/dish. Three cell counts for each cell line were made every $24 \mathrm{~h}$ for 10 days. Cell growth curves were made with cell number for ordinate and time for abscissa. Doubling time $\left(\mathrm{T}_{\mathrm{d}}\right)$ was calculated based on the formula: $\mathrm{T}_{\mathrm{d}}=\mathrm{T} \times \lg 2 / \lg \left(\mathrm{N}_{1} / \mathrm{N}_{0}\right) \cdot \mathrm{N}_{1}\left(\mathrm{~N}_{0}\right)$ stands for the cell number at $\mathrm{T}_{1}\left(\mathrm{~T}_{0}\right)$ time during logarithmic growth phase. $\mathrm{T}=\mathrm{T}_{1}-\mathrm{T}_{0}$.

3-(4,5-dimethylthiazol-2-yl)-2,5-diphenyltetrazolium bromide (MTT) assay. Cells were seeded at the amount of $5 \times 10^{3} /$ well at 96-well tissue culture plates. After $12 \mathrm{~h}$ of incubation, a series of drug concentration gradients were added to the wells, 6 repeats for one concentration. Sixty-nine hours later, MTT solution was added. Another $3 \mathrm{~h}$ later, the medium containing MTT was replaced with $150 \mu \mathrm{l}$ of DMSO in each well to dissolve the formazan crystals. The absorbance was detected at $560 \mathrm{nM}$ using a microplate reader (Bio-Rad, Sunnyvale, CA, USA).

Cell growth rate and drug resistance in vivo. To establish human breast xenografts, BCap37, BC-DS and BC-TS ( $0.2 \mathrm{ml}$ PBS containing $1 \times 10^{6}$ cells) were injected into the right armpits of the homozygous nude athymic mice (female, 5-6-weeks old). Each cell line had two groups. The control groups were treated with PBS, while the treatment groups were treated with vinorelbine $(5 \mathrm{mg} / \mathrm{kg}$, intraperitoneal injection). The injections were repeated every 6 days for 6 injection cycles. Width (a) and length (b) of the tumors were measured every 3 days. Tumor volume was calculated with the formula: $\mathrm{V}=(\pi / 6) \times \mathrm{ab}^{2}$. When the mice were terminated, the tumor tissues were removed and weighted. Data are representative of two separate experiments.

Western blotting. Cellular proteins were prepared with a protein lysis buffer (Beyotime, Haimen, China) and its concentration was measured by BCA protein assay kit (KeyGen Biotech, Nanjing, China). Equal samples (15 $\mu$ l containing $45 \mu \mathrm{g}$ ) of protein were separated on $6-10 \%$ SDS-PAGE gels and then transferred to polyvinylidene difluoride membranes. Then these membranes were blocked for $1 \mathrm{~h}$ at room temperature with 5\% non-fat dry milk in Tris-buffered saline $(150 \mathrm{mM}$ $\mathrm{NaCl}, 20 \mathrm{mM}$ Tris- $\mathrm{HCl}, \mathrm{pH}$ 7.5). Membranes were washed in PBST and respectively incubated with anti-MDR1, anti-tubulin primary antibodies (Santa Cruz Biotechnology, Santa Cruz, CA, USA) at $4^{\circ} \mathrm{C}$. After overnight incubation, the membranes were washed with PBST, and incubated with horseradish peroxidase-conjugated goat anti-mouse $\operatorname{IgG}$ followed by enhanced chemiluminescent staining using the ECL system. Tubulin was used for normalization of protein loading.

Cell cycle arrest assay. Three cell lines were treated with $20 \mathrm{nM}$ vinorelbine for $48 \mathrm{~h}$ and then harvested by trypsinization. After centrifugation, cells were fixed in $70 \%$ ethanol at $4{ }^{\circ} \mathrm{C}$ overnight and then resuspended in propidium iodide staining solution containing $20 \mathrm{mg} / \mathrm{ml}$ propidium iodide and $0.5 \mathrm{mg} / \mathrm{ml}$ RNase in PBS at room temperature for $30 \mathrm{~min}$ before analysis by flow cytometry. Flow cytometric analysis was performed with a Beckman Coulter flow cytometer (Beckman Coulter, Miami, FL, USA) with an excitation at $488 \mathrm{~nm}$ and an emission at $630 \mathrm{~nm}(15)$.

Annexin V/PI assay. Three cell lines were treated with $20 \mathrm{nM}$ vinorelbine for $48 \mathrm{~h}$ and then harvested by trypsinization. After centrifugation, cells were washed with PBS and incubated in the dark for $10 \mathrm{~min}$ at room temperature in $100 \mu \mathrm{l}$ binding buffer (10 mM HEPES, pH 7.4; $140 \mathrm{mM} \mathrm{NaCl}$; $2.5 \mathrm{mM} \mathrm{CaCl}_{2}$ ) (Beyotime) containing Annexin V-FITC (40 $\mu \mathrm{l} / \mathrm{ml})$ and PI $(1 \mu \mathrm{g} / \mathrm{ml})$. After incubation, $400 \mu \mathrm{l}$ binding buffer was added to each sample and cells were kept on ice (16). Flow cytometric analysis was performed with a Beckman Coulter flow cytometer (Beckman Coulter). The 488-nm laser was used for excitation and FITC was detected in FL-1 by a 525/30-bp filter while PI was detected in FL-2 by a 575/30-bp filter (16).

In vitro migration assay. Migration assays were performed in a 24-well Transwell chamber (Corning, Cambridge, MA, USA). Three cell lines were harvested at logarithmic growth phase and plated into upper chambers $(0.2 \mathrm{ml}$ serum-free medium containing $1 \times 10^{5}$ cells). The lower parts of the chambers were filled with $0.5 \mathrm{ml}$ of RPMI-1640 medium containing $10 \%$ FBS. After $24 \mathrm{~h}$ of incubation, the migration cells were stained and enumerated.

Rhodamine 123 efflux assay. Each cell line had four groups. The control groups were incubated with Rhodamine $123(10 \mu \mathrm{g} /$ $\mathrm{ml}$ ) for $30 \mathrm{~min}$. In treatment $1,3 \mathrm{~h}$ of incubation was added with $5 \mu \mathrm{M}$ verapamil before Rhodamine 123 . Treatment 2 was followed by $2 \mathrm{~h}$ of incubation with RPMI-1640 medium based on treatment 1 . Treatment 3 was followed by $2 \mathrm{~h}$ of incubation with $5 \mu \mathrm{M}$ verapamil based on treatment 1 . After washing with $4^{\circ} \mathrm{C}$ PBS, and harvesting by trypsinization, intracellular Rhodamine 123 fluorescence intensity was determined with Coulter Epics V instrument (Beckman Coulter, Fullerton, CA, USA).

Statistical analysis. Data are presented as mean \pm standard error of three independent experiments. Two-sided Student's t-test was used to determine the statistical difference between various experimental and control groups. Differences were considered statistically significant at $\mathrm{p}<0.05$ (17).

\section{Results}

Establishment and morphological characterization of $B C-D S$ and BC-TS. Two vinorelbine-resistant sublines BC-DS and 
A

BCap37 or BC-DS-X cells

$(X=2,5,10,20,50 \mathrm{nM}$ VNB)

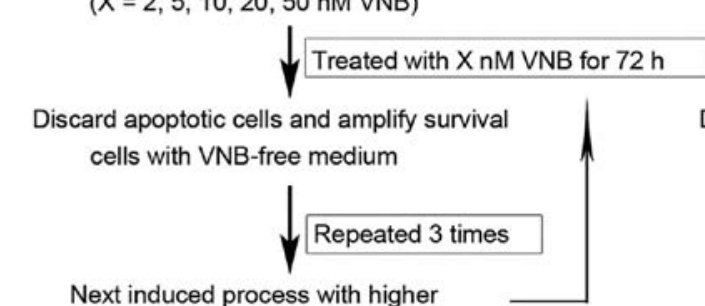

Next induced process with higher
concentration of VNB

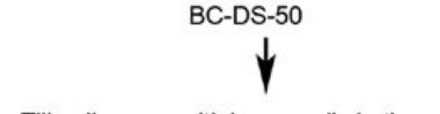

Till cells can multiply normally in the medium contains $50 \mathrm{nM}$ VNB

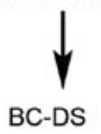

C

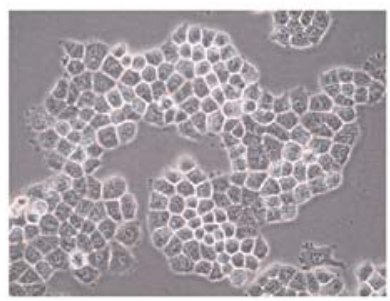

D
B

BCap37 or BC-TS-X cells $(X=1,2,4,8,12,24,48$ h) Treated with $50 \mathrm{nM}$ VNB for $\mathrm{Xh}$

Discard apoptotic cells and amplify survival cells with VNB-free medium

Next induced process with longer Repeated 3 times exposure times<smiles>[3H]</smiles>

BC-TS-48 $\downarrow$ Treated with $50 \mathrm{nM}$ VNB for $72 \mathrm{~h}$

Discard apoptotic cells and amplify survival cells with VNB-free medium

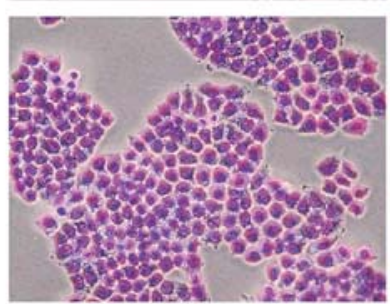

BCap37
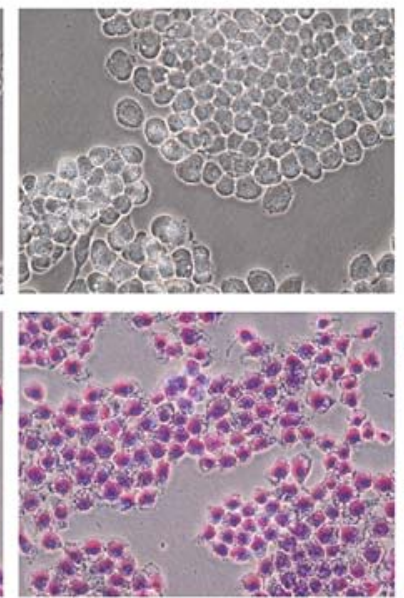

BC-DS
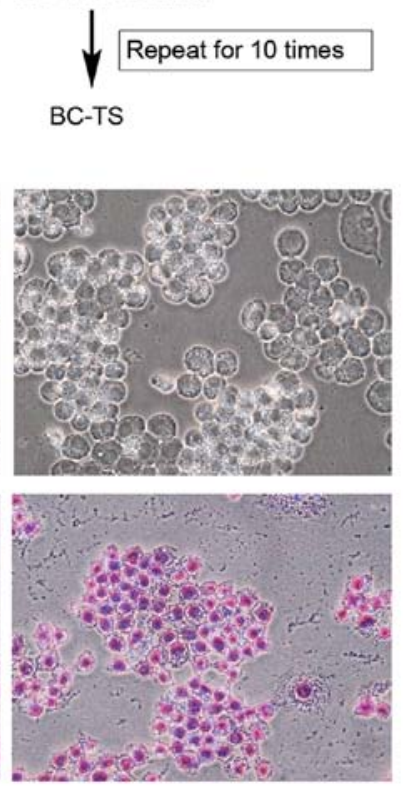

BC-TS

Figure 1. Establishment and morphological characterization of BC-DS and BC-TS. (A) BC-DS cells were selected based on continuous exposure to vinorelbine using a dose-stepwise incremental strategy. (B) BC-TS were selected based on a strategy of pulsed exposure to vinorelbine with time-stepwise increments. (C and D) Morphological characteristics were determined by inverted microscopic examination after Giemsa staining.

BC-TS, were successfully established from the human breast cancer cell line BCap37, with different 'two-stage screening methods'. BC-DS cells were selected based on continuous exposure to vinorelbine using a dose-stepwise incremental strategy (Fig. 1A). In the adaptation stage, BCap37 cells were exposed to vinorelbine from 2 to $50 \mathrm{nM}(2,5,10,20$, $50 \mathrm{nM}$ ) for $72 \mathrm{~h}$ step by step. In the consolidation stage, previously selected cells were continuously cultured in medium containing $50 \mathrm{nM}$ vinorelbine. When they multiplied normally, we had established the BC-DS cell line.

BC-TS were selected based on a strategy of pulsed exposure to vinorelbine with time-stepwise increments (Fig. 1B). In the adaptation stage, BCap37 cells were exposed to $50 \mathrm{nM}$ vinorelbine for 1 to $48 \mathrm{~h}(1,2,4,12,24,48 \mathrm{~h})$ step by step. In the consolidation stage, previously selected cells were exposured ten times to $50 \mathrm{nM}$ vinorelbine for $72 \mathrm{~h}$. The resulting cell line was named as BC-TS.
Morphological characterizations of BC-DS and BC-TS are different from the parental BCap37 cells (Fig. 1C and D). Bcap37 cells grew closely and had clearly demarcated colony edges while BC-DS and BC-TS had larger cell size and the cells grew loosely with various shapes.

The biological characterizations of BC-DS and BC-TS differed at different levels compared to the parental Bcap37. Biological characterization of breast cancer cells may change during the establishment of drug-resistant sublines. Thus, we examined the growth rate of BCap37, BC-DS and $\mathrm{BC}-\mathrm{TS}$ both in vitro and in vivo (Fig. $2 \mathrm{~A}$ and $\mathrm{B}$ ). Based on the data from in vitro growth assays, the doubling time of BCap37, BC-DS and BC-TS were 34.1 $\pm 2.3,43.8 \pm 3.3$ and $35.0 \pm 3.9 \mathrm{~h}$, respectively. Thus, BC-DS had slower proliferation rate compared to $\mathrm{BCap} 37$. While $\mathrm{BC}-\mathrm{TS}$ had exactly the same proliferation rate as BCap37, however, its 

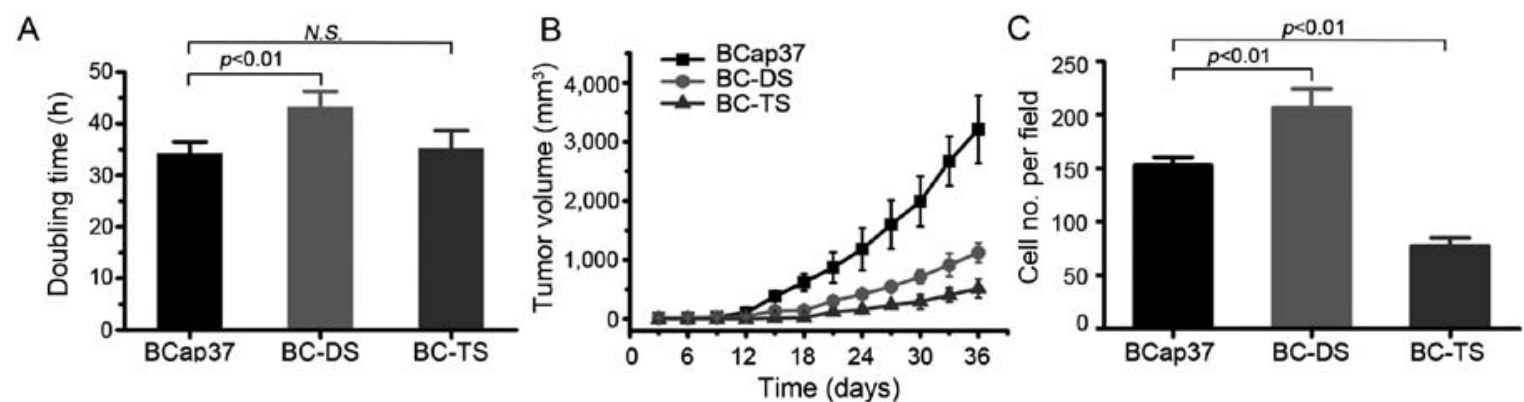

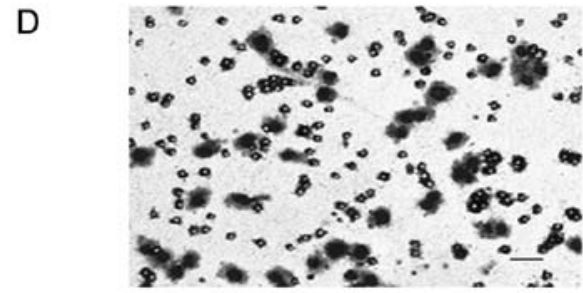

BCap37

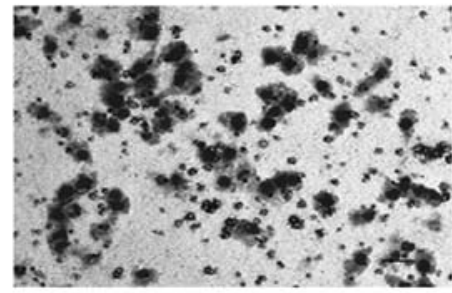

BC-DS

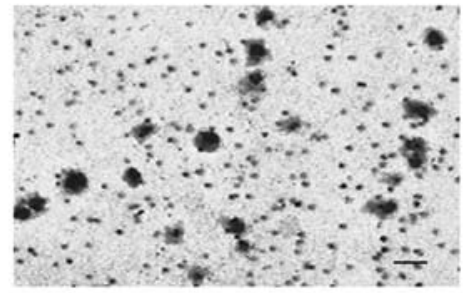

BC-TS

Figure 2. The biological characterizations of BC-DS and BC-TS. (A) The doubling time for BCap37, BC-DS and BC- TS cell lines. (B) Tumor volume curves of nude mice bearing BCap37, BC-DS and BC-TS. (C) Histogram showed the number of invasive cells per field. (D) Migration of BCap37, BC-DS and BC-TS were analyzed by Transwell assay. After $24 \mathrm{~h}$ incubation, the number of cells on the lower surface of the filters was quantified under a microscope. Bar, $20 \mu \mathrm{m}$. P-values were determined by one-way ANOVA test.

adaptability became worse, as it needed more time to reach logarithmic growth phase.

To study the proliferation ability of BCap37, BC-DS and BC-TS cells in vivo, we further established tumor xenograft models with homozygous nude athymic mice. In vivo, BCap37 still has the most aggressive proliferation based on the in vitro data, and BC-TS needed the longest time to adapt (Fig. 2B).

To analyze the migratory activity of BCap37, BC-DS and BC-TS cells, Transwell assay was conducted (Fig. 2C and D). We counted the migrated cells of these three cell lines. Compared with parental BCap37 cells, there were $35.3 \%$ more cells migrating successfully in BC-DS, but $48.4 \%$ less in $\mathrm{BC}-\mathrm{TS}$. The results indicate that the migration ability of BC-DS was enhanced, but that of BC-TS was attenuated.

$B C-D S$ and $B C-T S$ resist vinorelbine in vitro, but the resistant characterizations are both unstable. BC-DS and BC-TS cell lines were selected as vinorelbine-resistant. We used MTT assay to examine their sensitivity to vinorelbine in vitro. The $\mathrm{IC}_{50}$ value of $72 \mathrm{~h}$ vinorelbine exposure for BCap37, BC-DS and BC-TS was $2.3 \pm 0.4,729 \pm 100$ and $120 \pm 21 \mathrm{nM}$, respectively (Fig. 3A and Table I). Thus, BC-DS and BC-TS were about 317-fold and 52-fold more resistant to vinorelbine than the parental BCap37.

To observe if BC-DS and BC-TS had stable vinorelbine-resistant characterizations, we cultured the three cell types in vinorelbine-free medium and detected the $\mathrm{IC}_{50}$ values every 30 days. As presented in Fig. $3 \mathrm{~B}$, the $\mathrm{IC}_{50}$ values at $72 \mathrm{~h}$ vinorelbine exposure decreased markedly in BC-DS and BC-TS with time. It took about 90 days for them to lose the vinorelbine-resistance.

We also used flow cytometric analyses, which indicated that both $\mathrm{BC}-\mathrm{DS}$ and $\mathrm{BC}-\mathrm{TS}$ were much more resistant to vinorelbine-induced cell cycle arrest and apoptosis. The cell cycle of BCap37 was obviously arrested in $20 \mathrm{nM}$ vinorelbine for $48 \mathrm{~h}$, while BC-DS and BC-TS were slightly affected in the same conditions (Fig. 3C). Annexin V/PI assay showed the percentage of apoptotic cells in $\mathrm{BCap} 37$ was 23.34, while it was only 5.34 and 8.33 for BC-DS and BC-TS, respectively (Fig. 3D).

$B C-D S$ and BC-TS resist vinorelbine in vivo. Sensitivity of BC-DS and BC-TS to vinorelbine was also observed in vivo. Vinorelbine had dramatic inhibiting effect of tumor growth on BCap37 (Fig. 4A and B), but little on BC-DS (Fig. 4C and D) and BC-TS (Fig. 4E and F). Corresponding tissue sections were stained with $\mathrm{H} \& \mathrm{E}$ or for the proliferation marker Ki-67. Compared to BCap37 cells, fewer BC-DS and BC-TS cells exhibited vacuolization and apoptotic features (Fig. 4G), but more BC-DS and BC-TS cells were Ki-67 positive (Fig. 4H), which proved their resistance to vinorelbine in vivo.

$B C-D S$ and BC-TS exhibit different phenotypes of multidrug resistance. Acquired multidrug resistance (MDR) is the main mechanism of chemotherapeutic drug resistance. We next examined their sensitivity to other chemotherapeutic agents including paclitaxel, doxorubicin, methotrexate, 5-fluorouracil and cisplatin. The MTT assay showed BC-DS and BC-TS exhibited significantly higher resistance than BCap37 to vinorelbine, paclitaxel, doxorubicin and cisplatin (Table I). As to 5-fluorouracil, there was a slight increase, but not significant in drug resistance on both BC-DS and BC-TS, whereas to methotrexate, $\mathrm{BC}$-DS became more sensitive while $\mathrm{BC}-\mathrm{TS}$ stayed the same. These findings suggested that BC-DS and BC-TS may represent two distinct MDR phenotypes.

$B C$-DS and BC-TS express P-glycoprotein $(P$-gp) at different level. Multidrug transporter P-gp could induce multidrug resistance after exposure to any drug tested (18). To determine 

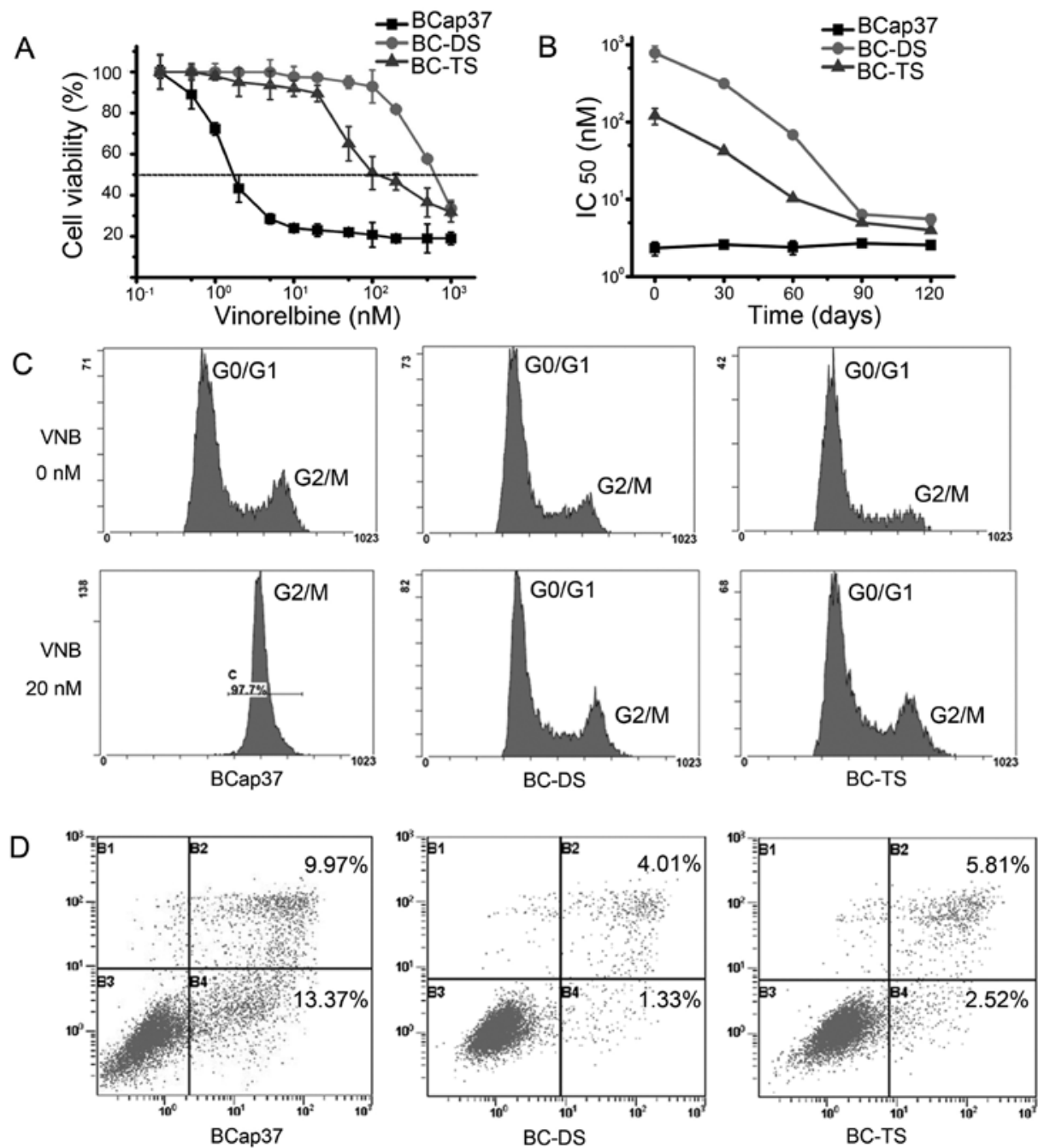

Figure 3. BC-DS and BC-TS resist vinorelbine in vitro, but are not stable. (A) MTT assay showed BC-DS and BC-TS resisted vinorelbine in vitro. Cells were treated with vinorelbine for $72 \mathrm{~h}$. (B) Both BC-DS and BC-TS had unstable vinorelbine-resistant characterization. $\mathrm{IC}_{50}$ values of $72 \mathrm{~h}$ vinorelbine exposure were evaluated by MTT assays every 30 days for BC-DS and BC-TS. (C) Both BC-DS and BC-TS were much more resistant to vinorelbine-induced cell cycle arrest and (D) apoptosis. Cells were treated with $20 \mathrm{nM}$ vinorelbine for $48 \mathrm{~h}$.

Table I. Drug sensitivity of BCap37, BC-DS and BC-TS.

\begin{tabular}{|c|c|c|c|c|c|}
\hline \multirow[b]{2}{*}{ Drug } & \multirow{2}{*}{$\frac{\text { BCap37 }}{\mathrm{IC}_{50}(\mathrm{nM})^{\mathrm{a}}}$} & \multicolumn{2}{|c|}{ BC-DS } & \multicolumn{2}{|c|}{ BC-TS } \\
\hline & & $\mathrm{IC}_{50}(\mathrm{nM})$ & $\mathrm{RI}^{\mathrm{b}}$ & $\mathrm{IC}_{50}(\mathrm{nM})$ & RI \\
\hline Vinorelbine & $2.3 \pm 0.4$ & $729 \pm 100^{\mathrm{d}}$ & 316.96 & $120 \pm 21^{\mathrm{d}}$ & 52.17 \\
\hline Paclitaxel & $4.1 \pm 0.2$ & $701 \pm 73^{d}$ & 170.98 & $85 \pm 7^{d}$ & 20.73 \\
\hline Doxorubicin & $231.6 \pm 19.7$ & $1,354 \pm 76^{\mathrm{d}}$ & 5.85 & $412 \pm 18.2^{c}$ & 1.78 \\
\hline Methotrexate & $18.2 \pm 0.8$ & $4.3 \pm 0.9^{\mathrm{d}}$ & 0.24 & $17.1 \pm 0.2$ & 0.94 \\
\hline 5-Fluorouracil & $9,144 \pm 945$ & $16,850 \pm 2,616$ & 1.84 & $11,380 \pm 593$ & 1.24 \\
\hline Cisplatin & $1,097 \pm 77$ & $1,755 \pm 148^{c}$ & 1.60 & $1,678 \pm 83^{c}$ & 1.53 \\
\hline
\end{tabular}

${ }^{\mathrm{a}} \mathrm{The}_{\mathrm{IC}}$ values were defined as the concentration of cells inhibiting growth at 50\%; b drug resistance index (RI) was determined by dividing $\mathrm{IC}_{50}$ values of BC-DS or BC-TS by that of BCap37 cells; ${ }^{\mathrm{c}} \mathrm{p}<0.05$, BC-DS or BC-TS vs BCap37; ${ }^{\mathrm{p}} \mathrm{p}<0.01$, BC-DS or BC-TS vs BCap37.

whether P-gp was one of the main reasons responding for multidrug resistance of BC-DS and BC-TS in our study, we detected its expression in three cell lines through western blotting. Compared with BCap37, BC-DS had a remark- able increase in P-gp expression while it was slight for BC-TS (Fig. 5A). This finding may explain the ability of BC-DS cells to tolerate a much higher concentration of vinorelbine than BC-TS. 
A

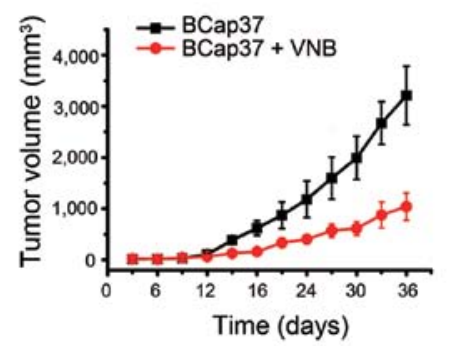

B

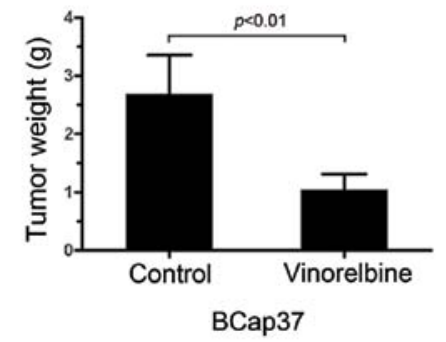

G
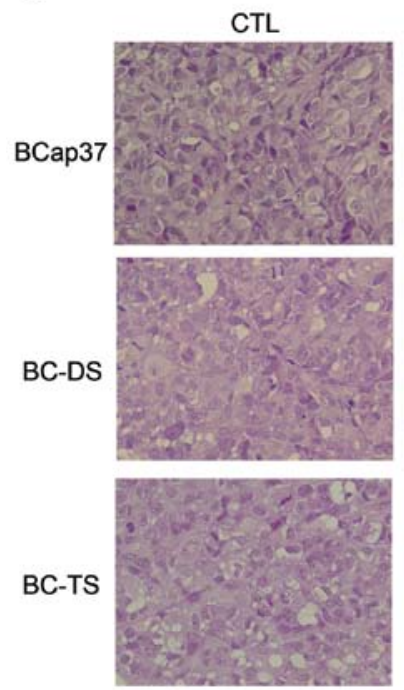

C

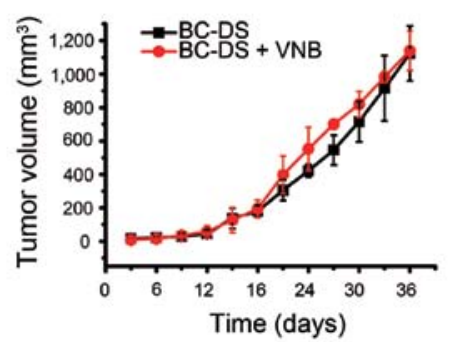

D

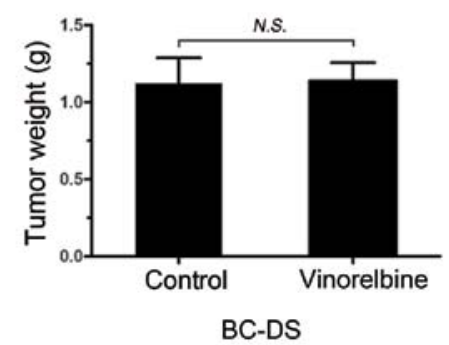

$\mathrm{E}$

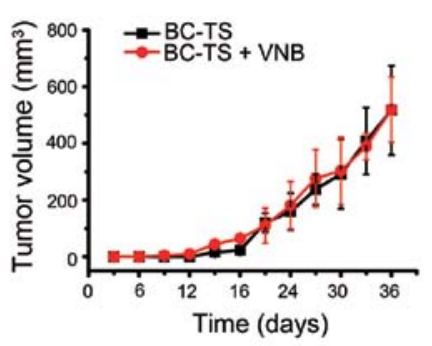

$\mathrm{F}$

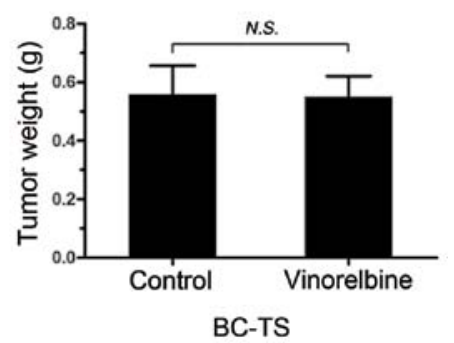

VNB
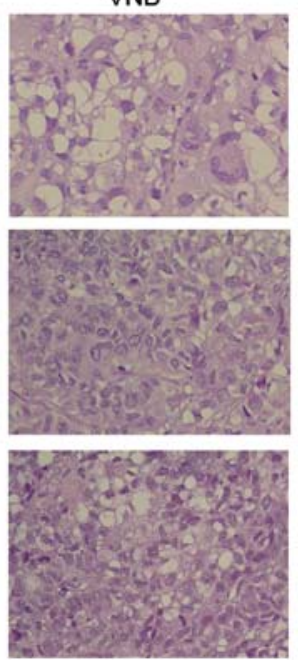

$\mathrm{H}$
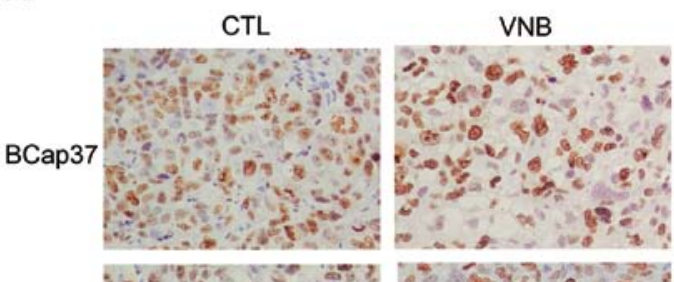

BC-DS
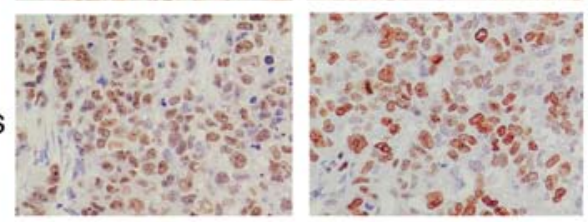

BC-TS
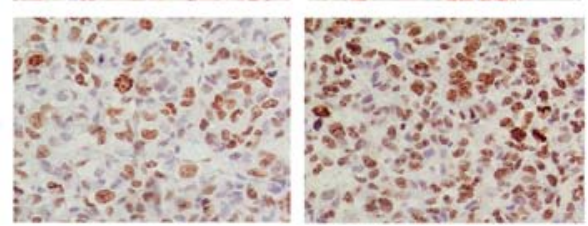

Figure 4. BC-DS and BC-TS resist vinorelbine in vivo. (A) BCap37 was sensitive to vinorelbine, both tumor volume and (B) weight were reduced when treated with vinorelbine. (C and D) BC-DS was significantly resistant to vinorelbine in vivo. (E and F) BC-TS was significantly resistant to vinorelbine in vivo. (G) Representative histological features of BCap37, BC-DS or BC-TS tumors treated with vehicle or vinorelbine. (H) Immunohistochemical staining of proliferation marker Ki-67 in BCap37, BC-DS or BC-TS tumors treated with vehicle or vinorelbine. Nude mice bearing BCap37, BC-DS or BC-TS tumors were treated with or without vinorelbine. P-values were determined by t-test.

Furthermore, to investigate whether intracellular drug accumulation was significantly decreased in BC-DS and $\mathrm{BC}-\mathrm{TS}$, Rhodamine 123 was used as a molecular probe in drug efflux assay. Verapamil is a calcium channel blocker and also a P-gp inhibitor that can reverse MDR (19). According to the assay (Fig. 5B), no significant change in Rhodamine 123 retention was observed in BCap37 cells with or without verapamil co-treatment. On the contrary, verapamil significantly inhibited Rhodamine 123 efflux in both BC-DS and BC-TS cell lines. Furthermore, quantity of Rhodamine 123 changed more in BC-DS than BC-TS before and after verapamil co-treatment, which indirectly indicated greater expression of $\mathrm{P}$-gp in the BC-DS cell line.

Interestingly, there were also unknown bands observed around $250 \mathrm{kDa}$, the concentration of which was similar to
P-gp, the expression was the most in BC-DS and the least in BCap37 (Fig. 5A), temporarily, it was named M250. The tight connection between M250 and P-gp strongly indicated M250 to be a potential tumor resistance-associated protein similar to P-gp.

\section{Discussion}

In this study, we have successfully established two vinorelbineresistant sublines, BC-DS and BC-TS, from the human breast cancer cell line BCap37, with different 'two-stage screening methods'.

Compared to the parental BCap37 cells, both BC-DS and BC-TS were less active, which was consistent with other literature $(20,21,12)$. While BC-DS and BC-TS could resist 
A
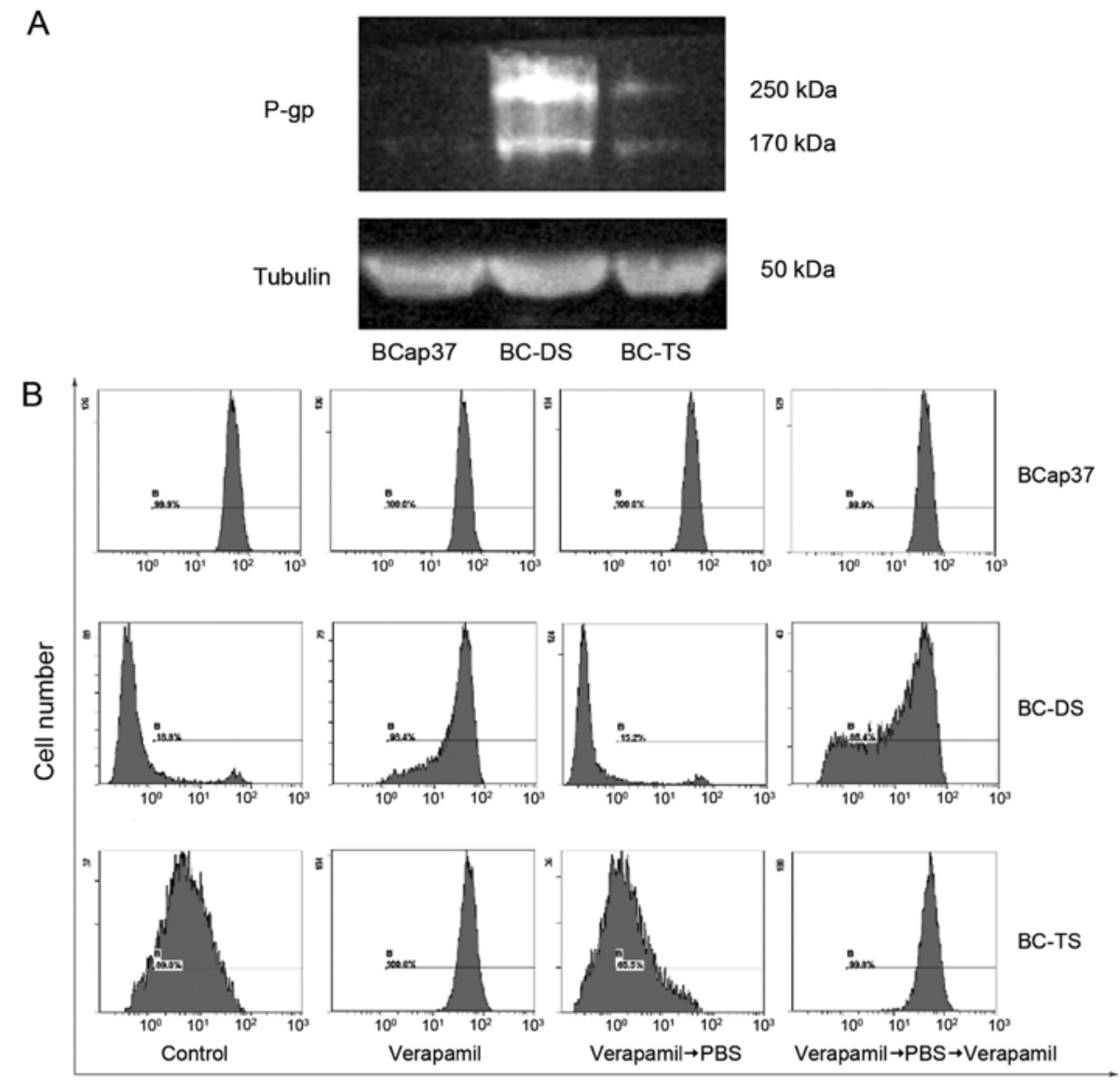

Fluorescence intensity

Figure 5. BC-DS and BC-TS express P-glycoprotein (P-gp) at different levels. (A) Detection of the expression of P-gp and tubulin in BCap37, BC-DS and BC-TS cells. Equal amounts ( $45 \mu \mathrm{g} / \mathrm{lane}$ ) of proteins were analyzed. (B) As described in methods, BCap37, BC-DS and BC-TS cells were treated with $10 \mu \mathrm{g} / \mathrm{ml}$ Rhodamine first, then with or without verapamil. The intracellular fluorescence intensity of Rhodamine 123 was determined with Coulter Epics V instrument.

vinorelbine in vitro and in vivo, they also gained multidrug resistance to paclitaxel, doxorubicin and cisplatin. Other researchers also discovered multidrug-resistant phenomena while investigating chemotherapy-resistant cancer cell lines they established (22-24). Interestingly, our study also showed that BC-DS became more sensitive to methotrexate (MTX). As MTX is one of the first-line antineoplastic drugs for breast cancer with relatively low price for patient, combination of MTX and vinorelbine could be a new treatment strategy. However, only few investigations were previously reported demonstrating the strategy to be a well-tolerated and effective regimen for patients with advanced breast cancer (25-27). Therefore, further research is needed to prove the safety and efficacy of this strategy.

MDR by increased efflux transporters, including ATP-binding cassette transporters is associated with upregulated $\mathrm{ABCB} 1$ expression and the main cause of treatment failure $(18,20,28)$, can be observed in the majority of cancers (29). The expression of P-gp was found to be upregulated strongly in BC-DS and slightly in BC-TS, which may result in the difference of their maximum tolerated concentration to chemotherapeutic agents. Felipe et al (10) reported that P-gp was overexpressed in the epirubicin-resistant gastric cancer line they established using dose-stepwise incremental strategy. Monoclonal antibody, antagonist or depleting agent against P-gp is promising to optimize the therapeutic effect of vinorelbine.
The results showed that vinorelbine-resistant characterization of both BC-DS and BC-TS were unstable. After being cultured in drug-free medium for two to three months, they became sensitive to vinorelbine again. Previous studies found cell lines established by dose-stepwise incremental strategy may be genetically unstable (11). Twentyman et al (20) adopted a pulsatile approach and found that the cell line was unstable during the first 3 weeks of drug-free growth, but with no loss of resistance if maintained in drug-containing media. However Jiang et al (17) reported that a cell line displayed stable resistant property using the pulsatile approach.

Moreover, different from BC-TS, BC-DS exhibited significantly enhanced migratory properties. It was also reported that drug-resistant cell line developed by time-stepwise increments administration exhibited enhanced migration (17). These contradictions may be due to the different drugs and parental cells used in the establishment, which suggested that different administration strategies with a single drug could induce distinct phenotypes of drug-resistant cell lines. It was the drugs and parental cells, not strategies that decided the final characterization of the produced variants.

BC-DS and BC-TS were distinct from each other and represented two different MDR phenotypes. Exposure may over time induce genetic events, which confer a drug-resistant phenotype on cells that were not intrinsically resistant at the start. Alternatively, resistant cells can be selected from 
a culture on the strength of an intrinsic mutation conferring resistance in that cell or group of cells, thus establishing them as the dominant clone in the culture (11). We speculate that BC-DS acquired drug-resistance, while BC-TS was intrinsically drug-resistant.

In clinical treatment, patients received vinorelbine intravenous $25-30$ or $60-80 \mathrm{mg} / \mathrm{m}^{2}$ orally in days 1 and 8 of a 21-day cycle (30-32), which was similar to the way we developed BC-TS cells. As discussed above, BC-TS shows lower migratory behavior and resistant ability compared to the conventional continuous exposure strategy developed in breast cancer BC-DS cells. In this aspect, our research showed that pulsed exposure is a better clinical medication strategy. Furthermore, the different phenotypes of multidrug resistance showed by BC-DS and BC-TS, are meaningful guidance for clinical drug combination.

BC-DS and BC-TS also provide opportunity for undertaking large-scale expression profile screening to identify novel biomarkers of chemotherapy resistance in breast cancer. The molecular weight of P-gp is $170 \mathrm{kDa}$, which is the most important MDR-associated protein (33). When we detected P-gp expression using western blotting, the 170-kDa bands appeared as we expected. However, there was also an unknown protein (M250), the concentration of which was similar to P-gp (Fig. 4A). This indicated M250 to be a potential tumor resistance-associated protein similar to P-gp. Part of our further research will focus on the mechanism and signal pathways of M250.

In summary, by using different screening strategies, we established two novel MDR cell lines, BC-DS and BC-TS, from chemo-sensitive human breast cancer cell line BCap37. Although BC-DS and BC-TS shared the same origin, they differed in many aspects. BC-TS cells show lower migratory behavior and resistant ability compared to the conventional continuous exposure strategy developed in breast cancer BC-DS cells, which verifies that pulsed exposure is a better clinical medication strategy. The unknown protein M250, we found in drug-resistant cancer cells, may be a potential tumor resistance-associated protein, which deserves further research.

\section{Acknowledgements}

This research was supported by grants NSFC-81372462, NSFC-81302288 and National Key Project on New Drug Developmental Program-2014ZX09507009026 (W. Fan).

\section{References}

1. Jemal A, Bray F, Center MM, Ferlay J, Ward E and Forman D: Global cancer statistics. CA Cancer J Clin 61: 69-90, 2011.

2. Xu YC, Wang HX, Tang L, Ma Y and Zhang FC: A systematic review of vinorelbine for the treatment of breast cancer. Breast J 19: 180-188, 2013.

3. Gregory RK and Smith IE: Vinorelbine - a clinical review. Br J Cancer 82: 1907-1913, 2000.

4. Calcagno AM, Fostel JM, To KK, Salcido CD, Martin SE, Chewning KJ, Wu CP, Varticovski L, Bates SE, Caplen NJ, et al: Single-step doxorubicin-selected cancer cells overexpress the ABCG2 drug transporter through epigenetic changes. Br J Cancer 98: 1515-1524, 2008.

5. Yang JX, Luo Y, Qiu HM and Tang WX: Characterization and resistance mechanisms of cisplatin-resistant human hepatocellular carcinoma cell line. Saudi Med J 30: 35-40, 2009.
6. Zhou Y, Ling XL, Li SW, Li XQ and Yan B: Establishment of a human hepatoma multidrug resistant cell line in vitro. World $\mathbf{J}$ Gastroenterol 16: 2291-2297, 2010.

7. Li L, Luan Y, Wang G, Tang B, Li D, Zhang W, Li X, Zhao J, Ding H, Reed E, et al: Development and characterization of five cell models for chemoresistance studies of human ovarian carcinoma. Int J Mol Med 14: 257-264, 2004.

8. Shin KH, Ku JL, Kim WH, Lee SE, Lee C, Kim SW and Park JG: Establishment and characterization of seven human renal cell carcinoma cell lines. BJU Int 85: 130-138, 2000.

9. Han T, Zhu X, Wang J, Zhao H, Ma Q, Zhao J, Qiu X and Fan Q: Establishment and characterization of a cisplatin-resistant human osteosarcoma cell line. Oncol Rep 32: 1133-1139, 2014.

10. Felipe AV, Moraes AA, de Oliveira J, da Silva TD and Forones NM: Establishment and partial characterization of an epirubicin-resistant gastric cancer cell line with upregulated ABCB1. Asian Pac J Cancer Prev 15: 6849-6853, 2014.

11. Watson MB, Lind MJ and Cawkwell L: Establishment of in-vitro models of chemotherapy resistance. Anticancer Drugs 18: 749-754, 2007.

12. Yang LY and Trujillo JM: Biological characterization of multidrug-resistant human colon carcinoma sublines induced/selected by two methods. Cancer Res 50: 3218-3225, 1990.

13. Ellis GK, Barlow WE, Gralow JR, Hortobagyi GN, Russell CA, Royce ME, Perez EA, Lew D and Livingston RB: Phase III comparison of standard doxorubicin and cyclophosphamide versus weekly doxorubicin and daily oral cyclophosphamide plus granulocyte colony-stimulating factor as neoadjuvant therapy for inflammatory and locally advanced breast cancer: SWOG 0012. J Clin Oncol 29: 1014-1021, 2011.

14. Pagliaro LC, Williams DL, Daliani D, Williams MB, Osai W, Kincaid M, Wen S, Thall PF and Pettaway CA: Neoadjuvant paclitaxel, ifosfamide, and cisplatin chemotherapy for metastatic penile cancer: A phase II study. J Clin Oncol 28: 3851-3857, 2010.

15. Wang W, Heideman L, Chung CS, Pelling JC, Koehler KJ and Birt DF: Cell-cycle arrest at G2/M and growth inhibition by apigenin in human colon carcinoma cell lines. Mol Carcinog 28: 102-110, 2000.

16. Foldbjerg R, Olesen P, Hougaard M, Dang DA, Hoffmann HJ and Autrup H: PVP-coated silver nanoparticles and silver ions induce reactive oxygen species, apoptosis and necrosis in THP-1 monocytes. Toxicol Lett 190: 156-162, 2009.

17. Jiang D, Sui M, Zhong W, Huang Y and Fan W: Different administration strategies with paclitaxel induce distinct phenotypes of multidrug resistance in breast cancer cells. Cancer Lett 335: 404-411, 2013.

18. Gottesman MM, Fojo T and Bates SE: Multidrug resistance in cancer: Role of ATP-dependent transporters. Nat Rev Cancer 2: 48-58, 2002.

19. Krishna R and Mayer LD: Multidrug resistance (MDR) in cancer. Mechanisms, reversal using modulators of MDR and the role of MDR modulators in influencing the pharmacokinetics of anticancer drugs. Eur J Pharm Sci 11: 265-283, 2000.

20. Twentyman PR, Fox NE, Wright KA and Bleehen NM: Derivation and preliminary characterisation of adriamycin resistant lines of human lung cancer cells. Br J Cancer 53: 529-537, 1986.

21. Yu DS, Ma CP and Chang SY: Establishment and characterization of renal cell carcinoma cell lines with multidrug resistance. Urol Res 28: 86-92, 2000.

22. Hour TC, Chen J, Huang CY, Guan JY, Lu SH, Hsieh CY and $\mathrm{Pu}$ YS: Characterization of chemoresistance mechanisms in a series of cisplatin-resistant transitional carcinoma cell lines. Anticancer Res 20: 3221-3225, 2000.

23. Uchiyama-Kokubu N and Watanabe T: Establishment and characterization of adriamycin-resistant human colorectal adenocarcinoma HCT-15 cell lines with multidrug resistance. Anticancer Drugs 12: 769-779, 2001.

24. Chung YM, Park S, Park JK, Kim Y, Kang Y and Yoo YD: Establishment and characterization of 5-fluorouracil-resistant gastric cancer cells. Cancer Lett 159: 95-101, 2000.

25. Abrahamova J, Wagnerova M, Kubala E, Malec V, Simova E, Sirakova I, Pavlikova E, Machova D, Kocak I, Pavlikova I, et al: Vinorelbine, epirubicin, and methotrexate (VEM) as primary treatment in locally advanced breast cancer. Oncologist 6: 347-352, 2001.

26. Elomaa I, Joensuu H and Blomqvist C: Vinorelbine, methotrexate and fluorouracil (VMF) as first-line therapy in metastatic breast cancer: A randomized phase II trial. Ann Oncol 14: 699-703, 2003. 
27. Subramanyan S, Abeloff MD, Bond SE, Davidson NE, Fetting JH, Gordon GB and Kennedy MJ: A phase I/II study of vinorelbine, doxorubicin, and methotrexate with leucovorin rescue as first-line treatment for metastatic breast cancer. Cancer Chemother Pharmacol 43: 497-502, 1999.

28. Richter M, Molnár J and Hilgeroth A: Biological evaluation of bishydroxymethyl-substituted cage dimeric 1,4-dihydropyridines as a novel class of P-glycoprotein modulating agents in cancer cells. J Med Chem 49: 2838-2840, 2006.

29. Cordon-Cardo C, O'Brien JP, Boccia J, Casals D, Bertino JR and Melamed MR: Expression of the multidrug resistance gene product (P-glycoprotein) in human normal and tumor tissues. J Histochem Cytochem 38: 1277-1287, 1990.

30. Ardavanis A, Tryfonopoulos D, Orphanos G, Ioannidis G, Karamouzis M and Rigatos G: First-line chemotherapy with fluorouracil-epirubicin-navelbine (FEN) combination in advanced breast cancer. Anticancer Res 25: 4493-4498, 2005.
31. Wang J, Xu B, Yuan P, Ma F, Li Q, Zhang P, Cai R, Fan Y, Luo Y and Li Q: Capecitabine combined with docetaxel versus vinorelbine followed by capecitabine maintenance medication for first-line treatment of patients with advanced breast cancer: Phase 3 randomized trial. Cancer 121: 3412-3421, 2015.

32. Stravodimou A, Zaman K and Voutsadakis IA: Vinorelbine with or without trastuzumab in metastatic breast cancer: A retrospective single institution series. ISRN Oncol 2014: 289836 , 2014.

33. Breier A, Barancík M, Sulová Z and Uhrík B: P-glycoprotein - implications of metabolism of neoplastic cells and cancer therapy. Curr Cancer Drug Targets 5: 457-468, 2005. 